\title{
STABILIZATION OF NONLINEAR SYSTEMS WITH PARAMETRIC UNCERTAINTY USING VARIABLE STRUCTURE TECHNIQUES ${ }^{1}$
}

D. A. Schoenwald

Instrumentation and Controls Division

Oak Ridge National Laboratory

P. O. Box 2009

Oak Ridge, TN 37831-8066
Ü. Özgüner

Department of Electrical Engineering

The Ohio State University

2015 Neil Avenue

Columbus, OH 43210-1272

To be presented at the

IFAC Symposium on Nonlinear Control Systems Design

Tahoe City, California

June 25-28, 1995

\begin{abstract}
"The submitted manuscript has been authored by a contractor of the U.S. Government under contract No. DE-AC05-84OR21400.

Accordingly, the U.S. Government retains a nonexclusive, royalty-free license to publish or reproduce the published form of this contribution, or allow others to do so, for U.S. Government purposes."
\end{abstract}

\footnotetext{
${ }^{1}$ Research sponsored by the Laboratory Directed Research Program of Oak Ridge National Laboratory, managed for the Department of Energy by Martin Marietta Energy Systems, Inc., under Contract No. DE-AC05-84OR21400.
}

6. DISTRBUTION OF THSS DOCUMENT IS UNLMTRED 


\section{DISCLAIMER}

This report was prepared as an account of work sponsored by an agency of the United States Government. Neither the United States Government nor any agency thereof, nor any of their employees, make any warranty, express or implied, or assumes any legal liability or responsibility for the accuracy, completeness, or usefulness of any information, apparatus, product, or process disclosed, or represents that its use would not infringe privately owned rights. Reference herein to any specific commercial product, process, or service by trade name, trademark, manufacturer, or otherwise does not necessarily constitute or imply its endorsement, recommendation, or favoring by the United States Government or any agency thereof. The views and opinions of authors expressed herein do not necessarily state or reflect those of the United States Government or any agency thereof. 


\section{DISCLAIMER}

Portions of this document may be illegible in electronic image products. Images are produced from the best available original document. 


\title{
STABILIZATION OF NONLINEAR SYSTEMS WITH PARA- METRIC UNCERTAINTY USING VARIABLE - STRUC- TURE TECHNIQUES*
}

\author{
D. A. SCHOENWALD**and Ü. ÖZGÜNER ${ }^{* * *}$ \\ *Research sponsored in part by the U. S. Department of Energy under Contract DE-AC05- \\ 84OR21400 with Martin Marietta Energy Systems, Inc. \\ **Instrumentation and Controls Division, Oak Ridge National Laboratory, P. O. Box 2009, Oak \\ Ridge, TN 37831-8066 U.S.A. E-Mail: schoenwaldda@ornl.gov \\ ***Department of Electrical Engineering, The Ohio State University, 2015 Neil Avenue, Columbus, \\ OH 43210-1272 U.S.A. E-Mail: umit@ee.eng.ohio-state.edu
}

\begin{abstract}
We present a result on the robust stabilization of a class of nonlinear systems exhibiting parametric uncertainty. We consider feedback linearizable nonlinear systems with a vector of unknown constant parameters perturbed about a known value. A Taylor series of the system about the nominal parameter vector coupled with a feedback linearizing control law yields a linear system plus nonlinear perturbations. Via a structure matching condition, a variable structure control law is shown to exponentially stabilize the full system. The novelty of the result is that the linearizing coordinates are completely known since they are defined about the nominal parameter vector, and fewer restrictions are imposed on the nonlinear perturbations than elsewhere in the literature.
\end{abstract}

Key Words. Exponentially stable; feedback linearization; nonlinear systems; uncertain dynamic systems; variable structure control

\section{INTRODUCTION}

In this paper, we consider the robust control of nonlinear systems that exhibit parametric uncertainty via feedback linearization and variable structure control techniques. The principal difficulty with feedback linearization is that the system dynamics must be known exactly for the nonlinearities to be successfully cancelled. Uncertainty in the system will result in residual nonlinear terms which will make the stabilization of the system more difficult. Uncertainty in nonlinear systems can arise from many sources: parametric uncertainty, unmodeled dynamics, computational errors, implementation details (e.g. sampling), etc. We consider the first of these sources.

In this paper, we follow a Lyapunov based approach, but we employ a unique parameterization of the system which allows some variable structure control techniques to be employed (Utkin, 1977; Young, 1978). Earlier work based on this approach appears in (Schoenwald and Özgüner, 1992; Schoenwald and Özgüner, 1994). One of the key differences between this work and that in (Spong and Sira-Ramirez, 1986; Spong and Vidyasagar, 1989) is that we show exponential stability is possible instead of just uniform boundedness. We start by expanding the state space equation of the uncertain system about the nominal parameter vector. Next, we linearize the nominal system (which is assumed to be linearizable) which results in a linear subsystem with higher order nonlinear terms. The linearizing coordinates are completely known (assuming the original state is measurable). Furthermore, the upper bound on the uncertain residual terms is much easier to obtain in this framework since this bound can be expressed directly in terms of parameter variations.

This is intuitively appealing for sensitivity analysis studies in which one is interested in examining the stability of a system under specific relative parameter variations. We also require a matching condition, but only the nominal system need be linearizable. An example is presented to illustrate the control method of this paper.

\section{PROBLEM STATEMENT}

Consider the single-input, single-output (SISO) nonlinear model

$$
\begin{aligned}
\dot{x} & =f(x, \alpha)+g(x, \alpha) u \\
y & =h(x)
\end{aligned}
$$

where it is assumed that $f(x, \alpha)$, and $g(x, \alpha)$ are $C^{\infty}$ vector fields defined on a dense submanifold $M \subset R^{n}$. It is also assumed that $h(x)$ is $C^{\infty}$ on 
$M$. In addition, $x$ is defined on an open set $U$ of $R^{n}$. The vector $\alpha$ is the unknown parameter vector. It is assumed that $f$ and $g$ are smooth vector fields for every $\alpha \in B_{\alpha} \subset R^{p}$ where $B_{\alpha}$ is an admissible set of unknown parameter vectors. Without loss of generality, we also assume that $f(0, \alpha)=0$ and $h(0)=0$. The nominal parameter vector $\alpha_{n}$ is assumed known and the perturbations about $\alpha_{n}$ are represented as

$$
\alpha=\alpha_{n}+\delta \alpha .
$$

Furthermore, the vector fields $f$ and $g$ are analytic in $\alpha$ about $\alpha=\alpha_{n}$.

Our goal is to find a diffeomorphism $\Phi(x)$ on $M$ and nonlinear functions $a(x)$ and $b(x)$ such that the nominal system is rendered linear in the coordinates $z=\Phi(x)$. That is,

$$
\begin{aligned}
\frac{\partial \Phi(x)}{\partial x}\left[f\left(x, \alpha_{n}\right)\right. & + \\
\left.g\left(x, \alpha_{n}\right) a(x)\right]_{x=\Phi^{-1}(z)} & =A z \\
\frac{\partial \Phi(x)}{\partial x}\left[g\left(x, \alpha_{n}\right) b(x)\right]_{x=\Phi^{-1}(z)} & =B
\end{aligned}
$$

with the nonlinear feedback

$$
u=a(x)+b(x) v .
$$

This results in the linear plant

$$
\begin{aligned}
\dot{z} & =A z+B v \\
y & =C z
\end{aligned}
$$

where $(A, B, C)$ are in the Brunovsky canonical form Brunovsky (1970) (see Isidori (1989) for a treatment on feedback linearization).

The input $v$ will not only be utilized to stabilize the nominal linear system (which has all its poles at the origin), but it will be used to exponentially stabilize the system once the parametric uncertainty is introduced. In the following sections, we define the Taylor series expansion about $\alpha_{n}$ and the control approach to stabilizing the uncertain system. In this paper, all vector norms are assumed to be the usual Euclidean norm, and all matrix norms are consistent with this vector norm.

\section{MODELING OF THE PARAMETRIC UNCERTAINTY}

We begin by expanding $\dot{x}$ as a Taylor series in $\alpha$ about $\alpha=\alpha_{n}$

$$
\begin{aligned}
\dot{x} & =f\left(x, \alpha_{n}\right)+g\left(x, \alpha_{n}\right) u \\
& +\left.\left(\frac{\partial f}{\partial \alpha}+\frac{\partial g}{\partial \alpha} u\right)\right|_{\alpha=\alpha_{n}} \delta \alpha
\end{aligned}
$$

$$
\begin{aligned}
& +\left.\frac{1}{2} \delta \alpha^{T}\left(\frac{\partial^{2} f}{\partial \alpha^{2}}+\frac{\partial^{2} g}{\partial \alpha^{2}} u\right)\right|_{\alpha=\alpha_{n}} \delta \alpha \\
& +\mathrm{O}\left(\|\delta \alpha\|^{3}\right)
\end{aligned}
$$

where it is noted that we have made no assumption regarding the linearity (or nonlinearity) of the uncertain parameters. We do, however, need the following assumption.

Assumption 1 The relative degree of the nominal system

$$
\begin{aligned}
& \dot{x}=f\left(x, \alpha_{n}\right)+g\left(x, \alpha_{n}\right) u \\
& y=h(x)
\end{aligned}
$$

is $r=n$ locally about some point $x=x^{0}$.

Assumption 1 implies that the locally diffeomorphic state transformation

$$
z=\Phi\left(x, \alpha_{n}\right)=\left[\begin{array}{c}
h(x) \\
L_{f\left(x, \alpha_{n}\right.} h(x) \\
\vdots \\
L_{f\left(x, \alpha_{n}\right)}^{r-1} h(x)
\end{array}\right]
$$

and the standard linearizing feedback

$$
\begin{aligned}
u & =a\left(x, \alpha_{n}\right)+b\left(x, \alpha_{n}\right) v \\
& =\frac{-L_{f\left(x, \alpha_{n}\right)}^{r} h(x)}{L_{g\left(x, \alpha_{n}\right)} L_{f\left(x, \alpha_{n}\right)}^{r-1} h(x)} \\
& +\frac{1}{L_{g\left(x, \alpha_{n}\right)} L_{f\left(x, \alpha_{n}\right)}^{r-1} h(x)} v
\end{aligned}
$$

result in the perturbed system

$$
\begin{aligned}
\dot{z} & =A z+B v \\
& +\frac{\partial \Phi\left(x, \alpha_{n}\right)}{\partial x}\left(\frac{\partial f}{\partial \alpha}+\frac{\partial g}{\partial \alpha} a\left(x, \alpha_{n}\right)\right. \\
& \left.+\frac{\partial g}{\partial \alpha} b\left(x, \alpha_{n}\right) v\right)\left.\right|_{\substack{\alpha=\alpha_{n} \\
x=\Phi^{-1}(z)}} \delta \alpha \\
& +\mathrm{O}\left(\|\delta \alpha\|^{2}\right) \\
y & =C z
\end{aligned}
$$

where $L_{f} h(x)=\frac{\partial h}{\partial x} f$ denotes the Lie derivative of $h(x)$ along $f(x)$. With the exception of $\delta \alpha$ (which can easily be bounded once relative parameter deviation is considered), all terms in 11 are known since they are evaluated about $\alpha=\alpha_{n}$. If the system is linear in the uncertainty $\alpha$ then the expansion 11 will be exact to first order in $\delta \alpha$. We now make the following assumption.

Assumption 2 The parameter vector $\alpha$ appears linearly in 1 .

The above assumption makes the expansion in 7 exact to first order in $\delta \alpha$. However, the work of this paper can easily be extended to higher order terms in $\delta \alpha$ as suggested by the expansion in 7 be- 
cause all the terms in the expansion are evaluated about the nominal parameter vector $\delta \alpha_{n}$ and thus are known. Thus, the results of this paper can be applied to any parametric uncertainty that can be expressed in finite polynomic form.

\section{ROBUST STABILIZATION}

The first step in the stabilization of the true nonlinear model is to place the poles of the nominal linear system $(A, B, C)$ into the left-half plane. This can be done via linear quadratic regulator theory or pole placement methods and will not be further discussed here. Instead, we assume the existence of a constant $1 \times n$ matrix $K$ (which is easy to find since the pair $(A, B)$ is completely controllable) such that $A_{c}=A+B K$ is stable. Thus, we let $v=K z+\Delta v$ which results in the new model

$$
\begin{aligned}
\dot{z} & =A_{c} z+B \Delta v \\
& +\frac{\partial \Phi}{\partial x}\left(\frac{\partial f}{\partial \alpha}+\frac{\partial g}{\partial \alpha} a\left(x, \alpha_{n}\right)\right. \\
& \left.+\frac{\partial g}{\partial \alpha} b\left(x, \alpha_{n}\right) K z\right)\left.\right|_{\substack{\alpha=\alpha_{n} \\
x=\Phi^{-1}(z)}} \delta \alpha \\
& +\left.\frac{\partial \Phi}{\partial x} \frac{\partial g}{\partial \alpha} b\left(x, \alpha_{n}\right)\right|_{\substack{\alpha=\alpha_{n} \\
x=\Phi^{-1}(z)}} \Delta v \delta \alpha
\end{aligned}
$$

where $\Delta v$ is an additional control term to stabilize the residual nonlinearities. To be able to control the above model, we need a matching condition that guarantees that the uncertain nonlinear terms lie in the range space of the input. More formally, we need the following assumption.

Assumption $\mathcal{} \forall x \in M, \forall \alpha \in B_{\alpha}$, we require that $\left.\frac{\partial g}{\partial \alpha}\right|_{\alpha=\alpha_{n}} \delta \alpha \in \operatorname{Span}\left\{g\left(x, \alpha_{n}\right)\right\}$ and $\left.\frac{\partial f}{\partial \alpha}\right|_{\alpha=\alpha_{n}}$ $\delta \alpha \in \operatorname{Span}\left\{g\left(x, \alpha_{n}\right)\right\}$.

The above assumption simply states that the uncertainty in 1 is mapped to a reachable part of the input space when transformed by the nominal linearizing coordinates. This assumption is less restrictive than some in the literature due to the fact that the $\operatorname{Span}\left\{g\left(x, \alpha_{n}\right)\right\}$ depends only on the nominal value of the parameters whereas in some works (e.g. Spong and Sira-Ramirez (1986)) it will depend on the uncertainty. Furthermore, the second part of Assumption 3 can be relaxed by replacing it with a condition involving Lie brackets as outlined in Akhrif and Blankenship (1988). It is shown there that this condition involving Lie brackets is less restrictive than the above. We now rewrite 12 as

$$
\dot{z}=A_{c} z+B \Delta v+\eta(z, \Delta v)
$$

where higher order terms in $\delta \alpha$ have been ignored and

$$
\eta(z, \Delta v)=\frac{\partial \Phi}{\partial x}\left(\frac{\partial f}{\partial \alpha}+\frac{\partial g}{\partial \alpha} a\left(x, \alpha_{n}\right)\right.
$$

$$
\begin{aligned}
& \left.+\frac{\partial g}{\partial \alpha} b\left(x, \alpha_{n}\right) K z\right)\left.\right|_{\substack{\alpha=\alpha_{n} \\
x=\Phi^{-1}(z)}} \delta \alpha \\
& +\left.\frac{\partial \Phi}{\partial x} \frac{\partial g}{\partial \alpha} b\left(x, \alpha_{n}\right)\right|_{\substack{\alpha=\alpha_{n} \\
x=\Phi^{-1}(z)}} \delta \alpha \Delta v .
\end{aligned}
$$

Our next assumption concerns the bounds on the uncertainty term $\eta(z, \Delta v)$.

Assumption 4 We assume the existence of a function $\rho(z, t)$ such that $\|\Delta v\| \leq \rho(z, t)$ and $\|\eta\| \leq$ $\rho(z, t)$.

The function $\rho(z, t)$ is written explicitly as a function of time because it depends on the state $z$. From Assumption 4 and the triangle inequality, we obtain

$$
\begin{aligned}
& \rho(z, t)=\left[1-\left\|\frac{\partial \Phi}{\partial x} \frac{\partial g}{\partial \alpha} b\left(x, \alpha_{n}\right)\right\|_{\substack{\alpha=\alpha_{n} \\
x=\Phi-1 \\
1_{1}(z)}}\right. \\
& \left.\sup _{\alpha}\|\delta \alpha\|\right]^{-1} \| \frac{\partial \Phi}{\partial x}\left(\frac{\partial f}{\partial \alpha}\right. \\
& +\frac{\partial g}{\partial \alpha} a\left(x, \alpha_{n}\right)
\end{aligned}
$$

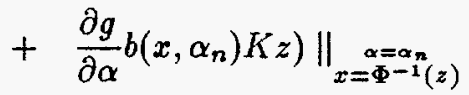

$$
\begin{aligned}
& \sup _{\alpha}\|\delta \alpha\| \text {. }
\end{aligned}
$$

It is important to note in 15 that everything on the right hand side is known a priori except for $\sup _{\alpha}\|\delta \alpha\|$ which is easily determined once one decides on a relative parameter deviation to consider. That is, we have expressed the uncertainty directly in terms of the parameter deviations which is physically more meaningful than if the parametric uncertainty were embedded within a complicated expression. For instance, if one wishes to determine the effects of $15 \%$ relative uncertainty for a scalar nonzero $\alpha$ then $\alpha\|\delta \alpha\|=$ $0.15 \alpha_{n}$. Our next assumption concerns the measurability of the state.

Assumption 5 The state $x$ (hence $z$ ) is available by direct measurement or via an observer.

The key idea is that since the transformed state $z$ is defined about the nominal parameters $\alpha_{n}$, it will be measurable if $x$ is measurable. The exponential stability proven in this paper is local due to the term in brackets in 15 possibly becoming unbounded. This determines the set $S$ of admissible initial conditions. From 15, the term in brackets must be bounded away from zero for all time to avoid a singularity. Since exponential stability will be proven in Theorem 1 , we only require this inequality to hold at time $t=t_{0}$. Under the conditions of the theorems, the inequality will then hold for all time. This is an advantage over (Spong and Sira-Ramirez, 1986; Spong and Vidyasagar, 1989) which can only claim uniform boundedness. 
Assumption 6 The inequality

$$
\left\|\frac{\partial \Phi}{\partial x} \frac{\partial g}{\partial \alpha} b\left(x, \alpha_{n}\right)\right\|\left\|_{\substack{\alpha=\alpha_{n} \\ x=\Phi^{-1}(z)}} \sup _{\alpha}\right\| \delta \alpha \|<1
$$

must hold at the initial conditions $z^{0}=\Phi\left(x^{0}\right)$ and $t=t_{0}$.

Additional assumptions made on the structure of $\rho(z, t)$ in (Spong and Sira-Ramirez, 1986; Spong and Vidyasagar, 1989) are not needed here which leads to a simpler control law to implement. The set of admissible initial conditions $S$ is then defined as

$$
\begin{aligned}
S= & \left\{z^{0} \in R^{n} \mid\right. \\
& \left\|\frac{\partial \Phi}{\partial x} \frac{\partial g}{\partial \alpha} b\left(x, \alpha_{n}\right)\right\|_{\substack{\alpha=\alpha_{n} \\
x^{0}=\Phi^{-1}\left(z^{0}\right)}} \\
& \left.\sup _{\alpha}\|\delta \alpha\|<1\right\} .
\end{aligned}
$$

The theorem that follows gives us the control law for $\Delta v$ and the stability result for the full system.

Theorem 1 Under Assumptions 1-6, the equilibrium $z=0$ of the system 13 is exponentially stable with respect to initial conditions lying in $S$ (defined in 17) if $\Delta v$ satisfies the variable structure control law

$$
\Delta v=\left\{\begin{array}{cc}
-\frac{\rho(z, t)}{\|B\|}, & z^{T} P B \geq 0 \\
\frac{\rho(z, t)}{\|B\|}, & z^{T} P B<0
\end{array}\right.
$$

where $\|B\|=1$ for the SISO case, and $P$ is the unique symmetric positive definite solution to the Lyapunov equation

$$
P A_{c}+A_{\varepsilon}^{T} P+Q=0
$$

with $Q$ a given symmetric positive definite matrix.

Proof: We first note that all terms in the above control law are completely known except for ${ }_{\alpha} \|$ $\delta \alpha \|$ which was discussed earlier. Further, the second part of Assumption $4(\|\Delta v\| \leq \rho(z, t))$ is satisfied by the above choice of control law. The proof follows some of the steps in the proof of Theorem 1 in Leitmann (1981), but we show exponential stability due to the different assumptions in this paper.

We start with the Lyapunov function for the nominal linearized system, $V(z)=z^{T} P z$. We proceed by showing that $V(z)$ is also a Lyapunov function for the system 13 with respect to the set $S$ in 17 . Differentiating along solutions of 13 and utilizing 19 , we obtain

$$
\begin{aligned}
\dot{V} & =2 z^{T} P A_{c} z+2 z^{T} P B \Delta v \\
& +2 z^{T} P \eta(z, \Delta v)
\end{aligned}
$$

$$
\begin{aligned}
& =-z^{T} Q z+2 z^{T} P B \Delta v+2 z^{T} P \eta(z, \Delta v) \\
& =-z^{T} Q z+2 z^{T} P B(\Delta v+\tilde{\eta}(z, \Delta v))
\end{aligned}
$$

where the last line is obtained from Assumption 3 (matching condition). This condition implies the existence of a function $\tilde{\eta}(z, \Delta v)$ such that

$$
\eta(z, \Delta v)=B \tilde{\eta}(z, \Delta v) .
$$

A bound is obtained on $\tilde{\eta}$ as follows.

$$
\|\eta\|=\|B \tilde{\eta}\|=|\tilde{\eta}|\|B\| \leq \rho(z, t)
$$

since $\tilde{\eta}$ is a scalar (SISO). From this we get

$$
-\frac{\rho(z, t)}{\|B\|} \leq \tilde{\eta} \leq \frac{\rho(z, t)}{\|B\|}
$$

which leads to

$$
\dot{V} \leq\left\{\begin{array}{c}
-z^{T} Q z+2 z^{T} P B(\Delta v \\
\left.+\frac{\rho(z, t)}{\|B\|}\right), z^{T} P B \geq 0 \\
-z^{T} Q z-2\left|z^{T} P B\right|(\Delta v \\
\left.-\frac{\rho(z, t)}{\|B\|}\right), \quad z^{T} P B<0
\end{array} .\right.
$$

With the choice of control law in 18 , we have

$$
\dot{V} \leq-z^{T} Q z \Longrightarrow \dot{V} \leq-\lambda_{\min }(Q)\|z\|^{2} .
$$

Since $Q$ is positive definite, all of its eigenvalues are positive. Thus, with reference to standard stability texts (e.g. Miller and Michel (1982), pp. 210-211), the equilibrium $z=0$ of 13 is exponentially stable subject to initial conditions lying in the set $S$ in 17. This completes the proof.

The control law above has a variable structure to it which as is shown in the proof above enables exponential stability to be proven. It should be noted that the control law in 18 is easier to implement than the control laws in (Leitmann, 1981; Spong and Sira-Ramirez, 1986; Spong and Vidyasagar, 1989). This is because the bound $\rho(z, t)$ is simpler to compute, since it can depend nonlinearly on $z$ instead of forcing one to find constants that make it a linear function of $z$ as in (Leitmann, 1981; Spong and Sira-Ramirez, 1986; Spong and Vidyasagar, 1989). Uniform boundedness of all solutions of 13 can be shown utilizing the same Lyapunov function. This might be useful in the event of more than one equilibrium point contained in the set $S$. A linear high gain control law is also possible to design. That is, there exists a large enough constant $\gamma>0$ such that $\Delta v=-\gamma B^{T} P z$ will make solutions of 13 uniformly ultimately bounded with respect to a different set $S$. The details of this approach are to be found in (Barmish et al., 1983; Thorp and Barmish, 1981). Assumption 5 can be relaxed (i.e. measurement noise) with only minor modifications by implementing some of the theory in 
(Barmish et al., 1983; Leitmann, 1981). Finally, the multi-input multi-output (MIMO) case requires some additional matrix manipulations, but the above result can still be extended to MIMO systems.

Example 1 We analyze the following second-order system

$$
\begin{aligned}
{\left[\begin{array}{c}
\dot{x}_{1} \\
\dot{x}_{2}
\end{array}\right] } & =\left[\begin{array}{c}
-x_{2} \\
x_{2} e^{-x_{1}}
\end{array}\right] \\
& +\left[\begin{array}{c}
0 \\
\alpha
\end{array}\right] u, \quad y=x_{1}
\end{aligned}
$$

which exhibits linear parametric uncertainty and has the origin as its equilibrium point. The nominal value of $\alpha$ is $\alpha_{n}=1$. The matching condition (Assumption 3) is also satisfied. We now consider the relative degree of the system

$$
\begin{aligned}
L_{g} h & =0 \\
L_{g} L_{f} h & =-\alpha \neq 0 .
\end{aligned}
$$

From above we see that for $\alpha=\alpha_{n}=1, r=$ $n=2$ as required in Assumption 1. This holds for a dense submanifold $M=R^{2}$ and the ball $B_{\alpha}=\{\alpha \in R \mid \alpha \neq 0\}$.

Continuing, we find that the linearizing coordinates and linearizing feedback in the nominal parameter is

$$
\begin{aligned}
z_{1} & =x_{1} \\
z_{2} & =-x_{2} \\
u & =-x_{2} e^{-x_{1}}-v .
\end{aligned}
$$

The Taylor series expansion of 26 about $\alpha=1$ in the new coordinates yields

$$
\begin{aligned}
\dot{z}_{1} & =z_{2} \\
\dot{z}_{2} & =v+\left(-z_{2} e^{-z_{1}}+v\right)(\alpha-1) \\
y & =z_{1}
\end{aligned}
$$

which is in the form of 11 . To determine the control law, we compute the bound $\rho(z, t)$. Substituting the above information and $\alpha=1$ into 15 , the bound

$$
\begin{aligned}
\rho(z, t) & =\left[1-\sup _{\alpha}|\alpha-1|\right]^{-1} \\
& \cdot\left|z_{2} e^{-z_{1}}+z_{1}+z_{2}\right|^{2} \cdot \sup _{\alpha}|\alpha-1|
\end{aligned}
$$

is obtained. In producing this bound, the gain vector $K=[-1-1]$ was chosen to place the poles of the nominal linearized plant in the open lefthalf plane. Since $\|B\|=1$, the control law is

$$
\Delta v=\left\{\begin{array}{cc}
-\rho(z, t), & \left(0.5 z_{1}+z_{2}\right) \geq 0 \\
\rho(z, t), & \left(0.5 z_{1}+z_{2}\right)<0
\end{array}\right.
$$

resulting in exponential stability of the origin. This was computed from 18 utilizing $P$ in 19 with $Q$ being the identity matrix.

Finally, the set of allowable initial conditions $S_{z}$ was calculated utilizing Assumption 6 and 28. It can be seen that $S=R^{2}$ provided that $\sup _{\alpha} \mid \alpha-$ $1 \mid<1 \forall \alpha \in B_{\alpha}$. That is, the equilibrium $x=0$ of 26 is globally exponentially stable with the control law 29 for nearly $100 \%$ relative uncertainty in $\alpha$ (i.e., $0<\alpha<2$ ).

\section{CONCLUSIONS}

We have shown that linearization and stabilization of nonlinear systems exhibiting parametric uncertainty is possible via a variable structure approach if certain assumptions (principally a structure matching condition) are satisfied. The principal advantages of the approach in this paper are that exponential stability instead of just uniform boundedness can be achieved and a control law that is easier to compute. In addition, the linearizing coordinates are known because they are defined about the nominal parameters, and systems without full relative degree can also be exponentially stabilized in this framework. Further, the bounds are expressed directly in terms of parametric uncertainty which makes them simple to compute once maximum parameter deviations are decided upon. The method avoids the feedback linearizability assumption of the whole family of uncertain plants as well as the convergence issues of an adaptive controller. The approach also works well with the ideas of sensitivity theory due to fact that the uncertainty is expressed directly in terms of the parameter deviations.

\section{ACKNOWLEDGEMENTS}

The authors wish to acknowledge the support of the AFOSR under Contract F49620-89-C-0046. The first author also gratefully acknowledges the support of the Ohio Aerospace Institute through a doctoral fellowship.

\section{REFERENCES}

Akhrif, O. and G. L. Blankenship (1988). Robust stabilization of feedback linearizable systems. In: Proceedings of the IEEE Conference on Decision and Control. Austin, TX. pp. 1714-1719.

Barmish, B. R., I. R. Petersen and A. Feuer (1983). Linear ultimate boundedness control of uncertain dynamical systems. Automatica 19, 523-532. 
Brunovsky, P. (1970). A classification of linear controllable systems. Kybernetika 6(3), 173188.

Isidori, A. (1989). Nonlinear Control Systems. second ed.. Springer-Verlag. Berlin.

Leitmann, G. (1981). On the efficacy of nonlinear control in uncertain linear systems. ASME Transactions on Dynamic Systems, Measurement and Control 102, 95-102.

Miller, R. K. and A. N. Michel (1982). Ordinary Differential Equations. Academic Press. New York.

Schoenwald, D. A. and Ü. Özgüner (1992). Robust feedback linearization of uncertain nonlinear systems. In: Proceedings of the American Contral Conference. Chicago, IL.

Schoenwald, D. A. and Ü. Özgüner (1994). Robust stabilization of nonlinear systems with parametric uncertainty. IEEE Transactions on Automatic Control AC-39(8), 1751-1755.

Spong, M. W. and H. Sira-Ramirez (1986). Robust control design techniques for a class of nonlinear systems. In: Proceedings of the American Control Conference. Seattle, WA. pp. 1515-1522.

Spong, M. W. and M. Vidyasagar (1989). Robot Dynamics and Control. John Wiley \& Sons. New York.

Thorp, J. S. and B. R. Barmish (1981). On guaranteed stability of uncertain linear systems via linear control. Journal of Optimization Theory and Applications 35(4), 559-579.

Utkin, V. I. (1977). Variable structure systems with sliding mode: A survey. IEEE Transactions on Automatic Control AC-22(2), 212222.

Young, K. K. D. (1978). Design of variable structure model-following control systems. IEEE Transactions on Automatic Control AC23, 1079-1085. 